\title{
Introduction to the Special Issue on End-to-End Speech and Language Processing
}

SPEECH AND LANGUAGE processing (SLP) is essentially a series of sequence-to-sequence learning problems. Conventional SLP systems map input to output sequences through module-based architectures where each module is independently trained. These have a number of limitations including local optima, assumptions about intermediate models and features, and complex expert knowledge driven steps. It can be difficult for non-experts to use and develop new applications. Integrated End-to-End (E2E) systems aim to simplify the solution to these problems through a single network architecture to map an input sequence directly to the desired output sequence without the need for intermediate module representations. E2E models rely on flexible and powerful machine learning models such as recurrent neural networks. The emergence of models for endto-end speech processing has lowered the barriers to entry into serious speech research. This special issue showcases the power of novel machine learning methods in end-to-end speech and language processing.

Following an open call for papers, we received a total of 41 submissions for this special issue, spanning a range of topics, including automatic speech recognition (ASR) and multimodal emotion recognition, information retrieval systems such as spoken keyword search, and text processing applications such as machine translation. After an extensive and competitive review process, we selected 11 papers for final publication.

Several papers of this special issue are devoted to ASR. There are two major types of E2E architectures for ASR; attention-based methods that use an attention mechanism to perform alignment between acoustic frames and recognized symbols, and connectionist temporal classification (CTC) that uses Markov assumptions to efficiently solve sequential problems by dynamic programming. S.
Watanabe et al. propose a hybrid CTC/attention end-to-end ASR, which effectively utilizes the advantages of both architectures for training and decoding. The hybrid approach allows the use of the forward-backward algorithm in CTC to enforce monotonic alignments and speed up the process of estimating the desired alignment, instead of solely depending on data-driven attention methods to estimate the desired alignments in long sequences.

While E2E approaches considerably simplify the build process for ASR systems, several challenges remain, including computational complexity, dealing with large sequences, and real-time performance. H. Tang et al. provide a combined review of neural segmental models from several prominent research groups. These models can be viewed as consisting of a neural network-based acoustic encoder and a finite-state transducer decoder. This study analyzes several weight functions that map the input acoustic features to the label sequence and loss functions that combine segmental and frame-level information for E2E training. The study draws connections between the various types of models, setting the stage for future research in ASR to benefit from its findings.

P. Doetsch et al. propose a novel, fully stochastic, inverted HMM architecture as an alternative to the CTC/attention-based E2E systems. This integrated discriminative model can be trained end-to-end from scratch by inversely aligning each element of an HMM state sequence to a segment-wise encoding of several consecutive input frames. In the inverted HMM, the HMM concept is generalized to drop the conditional independence assumption on the frame or label level while keeping the alignment as part of the underlying stochastic model

While all these approaches on E2E frameworks focus on the ASR problem in a single-channel setup without speech enhancement, T. Ochiai et al propose a unified architecture to incorporate a multi-channel setup with speech enhancement within an E2E framework. The proposed approach 
is a single, fully-differentiable, E2E neural network with a neural beamformer that can be optimized with a combined CTC and attention-based ASR objective. A similar integrated approach to jointly learn the front-end signal processing and back-end acoustic modeling of an ASR system is proposed by B. Wu et al. A joint speech dereverberation for signal enhancement and a deep neural network based acoustic modeling architecture is presented.

An end-to-end audio-visual emotion recognition system is proposed by P. Tzirakis et al. Instead of using hand-crafted features, a convolutional neural network $(\mathrm{CNN})$ is used to extract emotional features from speech and a deep residual network is used for vision. A long short-term memory (LSTM) neural network is then used to combine the automatically extracted audio and visual feature streams.

This Special Issue presents several articles on keyword search (KWS), which investigate the relationship between learning and different components of KWS systems. Fuchs and Keshet introduce a calibrated loss function that maximizes performance at a specific decision threshold, rather than simply trying to maximize the margin between positive and negative examples. They apply it to both classical phonetic queries and query-byexample (QbyE). Gündoğdu et al. jointly learn a trainable distortion measure for sub-sequence dynamic time warping and a model to map phone sequences with durations to pseudo-posteriorgrams, so that techniques from QbyE search can be used to find OOV queries in a system where queries are presented in textual format. In this case, both a loss function and a query representation are learned.

Chen et al. focus on the problem of keyword search in a low-resource setting where no transcribed speech is available in the target language. They propose using a multi-task learning (MTL) approach to train a bottleneck neural network feature extractor. The MTL network is trained to map features from the target language to phoneme-like units induced using a Dirichlet process Gaussian mixture model, and to map features from an auxiliary, high-resource language for which transcripts are available to HMM states.

The final KWS paper, by Audhkhasi et al., proposes an ASR-free KWS system built from three neural models that are trained jointly: an RNN acoustic encoder that maps variable-length feature sequences to a fixed-length embedding, a CNNRNN encoder that maps the text query to a fixedlength embedding, and a feedforward network that predicts the presence or absence of the query in the audio.

E2E neural machine translation has become stateof-the-art for language pairs with large amounts of parallel data. The paper by C. España-Bonet et al. focuses on multilingual translation over a set of languages (i.e., Arabic, English, French, German, and Spanish). They investigate the interlingual nature of the context vectors generated by their multilingual neural machine translation system and study their power in the assessment of monolingual and cross-language similarity. Four research questions are addressed: whether embeddings learned for a source text also depends on the target language, how distinguishable representations of semantically similar and semantically-distant sentence pairs are, how close representations of sentence pairs within and across languages are, and how representations evolve throughout the training.

Finally, we would like to thank all the authors and reviewers whose contributions have made this special issue possible. We would like to thank Prof. Shri Narayanan, Editor-in-Chief, for his support, and encouragement. Warmest thanks also to Allison Fleisher from the IEEE publication office for keeping the issue on track.

\author{
BHUVANA RAMABHADRAN, Lead Guest Editor \\ IBM T.J. Watson Research Center \\ Yorktown Heights, NY 10598, USA \\ NANCY F. CHEN, Guest Editor \\ Insititute for Infocomm Research (I2R) \\ A*STAR, Singapore \\ MARY P. HARPER, Guest Editor \\ Army Research Laboratory \\ Adelphi, MD 20783, USA \\ BRIAN KINGSBURY, Guest Editor \\ IBM T.J. Watson Research Center \\ Yorktown Heights, NY 10598, USA \\ KATE KNILL, Guest Editor \\ University of Cambridge \\ Cambridge CB2 1PZ, U.K.
}




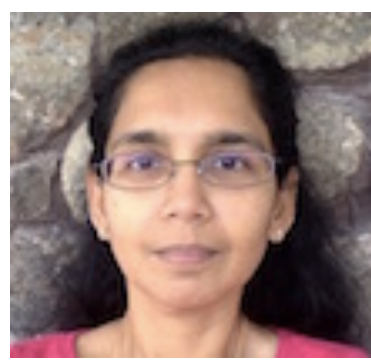

Bhuvana Ramabhadran (F'17) is a Distinguished Research Staff Member and Manager in IBM Research AI. She leads a team of researchers in the Speech Technologies Group and co-ordinates activities across IBM's world-wide labs in the areas of speech recognition, synthesis, and spoken term detection. She was the elected chair of the IEEE SLTC (20142016), area chair for ICASSP (2011-2017), Interspeech (2012-2016), on the editorial board of T-ASLP (2011-2015), and is currently an ISCA board member. She is a Fellow of ISCA.

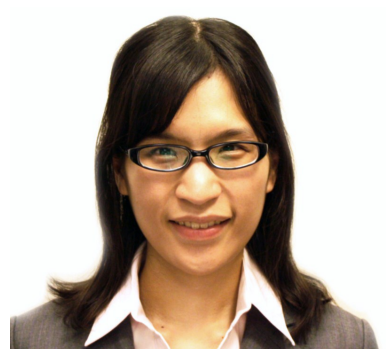

Nancy F. Chen received her $\mathrm{PhD}$ from MIT and Harvard (2011) based on her research at MIT Lincoln Laboratory in multilingual speech processing. She is currently leading initiatives in deep learning and human language technology at I2R, A*STAR, Singapore. She is an elected member of IEEE SLTC (20162018) and has received multiple awards, including Best Paper at APSIPA ASC (2016), the Microsoftsponsored IEEE Spoken Language Processing Grant (2011), and the NIH Ruth L. Kirschstein National Research Award (2004-2008).

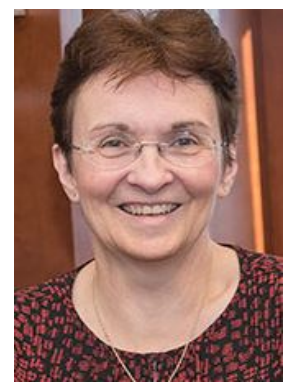

Mary P. Harper (SM'02) is Deputy Chief Scientist at Army Research Laboratory. She also has served as a Program Manager for the Babel Program at IARPA. She holds a Ph.D. in Computer Science from Brown University. She has served as a guest member of IEEE SLTC (2007-2010), on the editorial board of T-ASLP (2005-2009), on Flanagan Speech and Audio Processing Award Committee (2014-2016), and is currently an ISCA board member. She is a Fellow of ISCA.

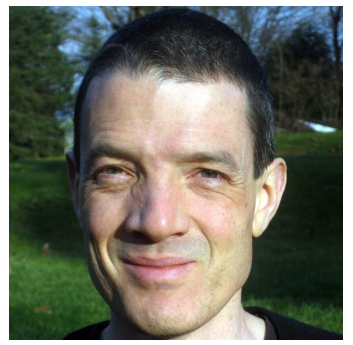

Brian Kingsbury (SM'09) is a principal research staff member in IBM Research AI.He has served on the IEEE SLTC (2009-2011); as an ICASSP speech area chair (2010-2012); an associate editor for IEEE Transactions on Audio, Speech, and Language Processing (2012-2016); and a program chair for the International Conference on Representation Learning (2014-2016). He holds a $\mathrm{PhD}$ in computer science from the University of California, Berkeley, and a BS in electrical engineering from Michigan State University.

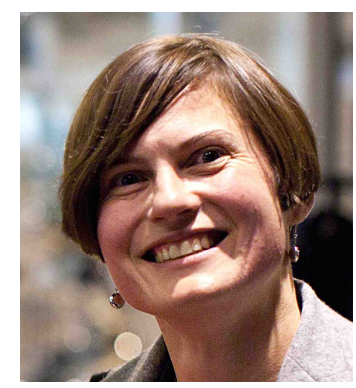

Kate Knill (M'01) is a Senior Research Associate at Engineering Department, Cambridge University, UK. She holds a $\mathrm{PhD}$ in Digital Signal Processing from Imperial College, London University, UK, and has over 25 years experience in speech and language processing in industry and academia, including the establishing and leading of Speech Technology Group, Toshiba Cambridge Research Lab, UK 2002-2012. She was a member of the IEEE SLTC 2009-2012, is an ISCA Board member (2013-2021) and is currently Secretary of ISCA. 